\title{
CLINICAL PRESENTATION OF PSORIATIC ARTHRITIS
}

\author{
R. SCARPA, R. PELUSO, M. ATTENO \\ Rheumatology Research Unit, University Federico II, Naples, Italy
}

\begin{abstract}
SUMMARY
Psoriatic arthritis is a spondyloarthropathy, which occurs in patients with skin and/or nail psoriasis. Basing its characterization on morphological purposes, several types of arthritis have been described. Alternatively, we propose a simplified classification into three subsets, focusing on the levels of expression of cutaneous and articular elements which devise this syndrome. The first is established psoriatic arthritis which occurs in patients with evident or remittent skin and/or nail psoriasis. Its clinical spectrum consists of the five subsets classically described by Moll and Wright in 1973. The second is psoriatic arthritis "sine psoriasis" which occurs in subjects without psoriasis but with a family history of the disease in first or second-degree relatives. The third is early psoriatic arthritis which consists of an articular involvement of recent onset, occurring in subjects belonging to established or sine psoriasis subsets.
\end{abstract}

Key words: Psoriatic arthritis, classification, clinical subsets

Psoriatic arthritis is a spondyloarthropathy, which occurs in patients with skin and/or nail psoriasis. Until today, rheumatologists have preferred to describe various aspects of its clinical presentation without focusing on the levels of expression of each element that devise this syndrome. Following this methodological approach, several types of psoriatic arthritis have been described. Alternatively to this approach, we believe that the real problem is not the description of all subsets of the disease, but a clinical characterization based on the levels of expression of each element which contribute to the composition of the syndrome (cutaneous and articular). Therefore, on the basis of our hypothesis, we suggest that three different forms of articular involvement should be considered in psoriatic patients:

1) established psoriatic arthritis, which occurs in subjects with evident or remittent skin and/or nail psoriasis;

2) psoriatic arthritis "sine psoriasis", which occurs in subjects without psoriasis but with a familial history of the disease in first or seconddegree relatives;

3) early psoriatic arthritis, consisting of an artic-

Corresponding author:

Raffaele Scarpa, MD

Cattedra di Reumatologia

Università di Napoli Federico II

Via S. Pausini 5 - 80131 Naples, Italy

E-mail: rscarpa@unima.it ular involvement of recent onset, occurring in subjects belonging to established or "sine psoriasis" subsets.

The three different forms of psoriatic arthritis are described in the following paragraphs.

\section{ESTABLISHED PSORIATIC ARTHRITIS}

This form may be considered as the classical psoriatic arthritis where cutaneous and articular elements are clearly expressed (Table I). In 1973, Wright and Moll described five subgroups which reflect all possible clinical presentations of this form (1). The precise composition and the relative frequency of these subgroups have since been the subject of debate because clinical patterns may change with time in individual patients. Indeed, Jones and co-workers in 1994 documented these pattern changes over time in over $60 \%$ of their patients with psoriatic arthritis (2). On the basis of our experience, spondylitis alone is the most frequent subset accounting for $30 \%$ of the case; it

Table I - Established psoriatic arthritis.

It is a spondyloarthropathy which occurs in subjects with evident or remittent skin and/or nail psoriasis.

Five clinical subsets may be its presentation.

In individual patients the clinical pattern may change over time (usually oligoarthritis becomes polyarthritis). 


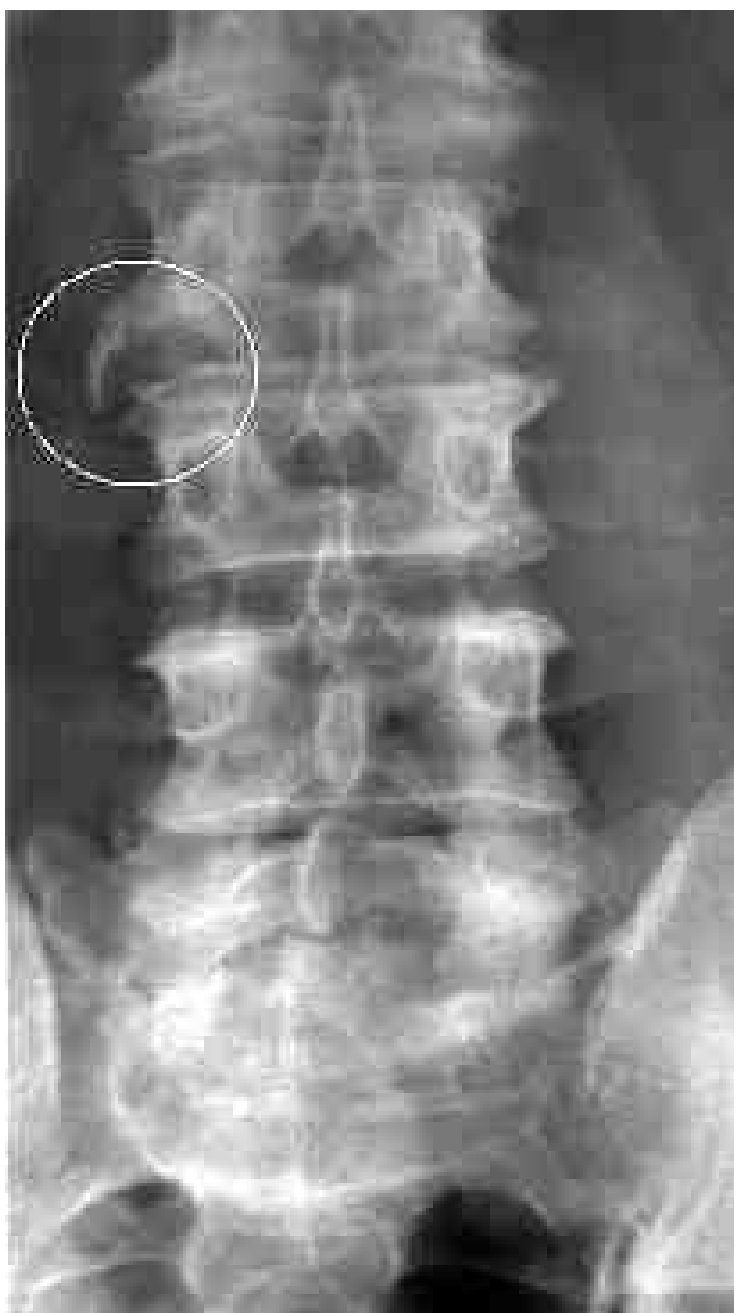

Figure 1 - Psoriatic spondylitis: asymmetrical marginal and nonmarginal syndesmophytes (white circle) randomly distributed along the lumbar tract of the spine. Observe the asymmetric sacroiliitis.

may overlap in another $30 \%$ with all peripheral subsets. Spinal changes, however, are less typical than those seen in ankylosing spondylitis. In fact, typical findings are the occurrence of unilateral sacroiliitis and non-marginal syndesmophytes randomly distributed along the spine (Fig. 1). In addition, the prevalence of HLA B27 is markedly low (3).

Polyarthritis is the second most frequent subset accounting for $20 \%$ of the cases. The distribution of synovitis may resemble that of the rheumatoid form (Fig. 2), but several aspects help differentiate these two entities (4). Apart from the usual absence of rheumatoid factors, joint involvement include: DIP involvement, anterior chest wall involvement, and the occurrence of enthesopathies

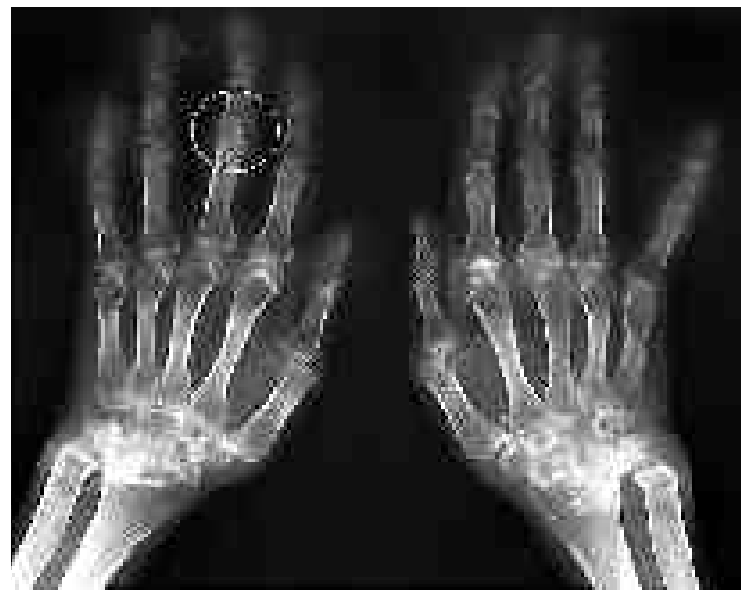

Figure 2 - Psoriatic symmetrical polyarthritis (rheumatoid-like form): severe involvement of wrist and metacarpophalangeal joints. In particular, observe consequence of "dactylitis", which at onset of arthritis involved third interphalangeal joint of the left hand (white circle).

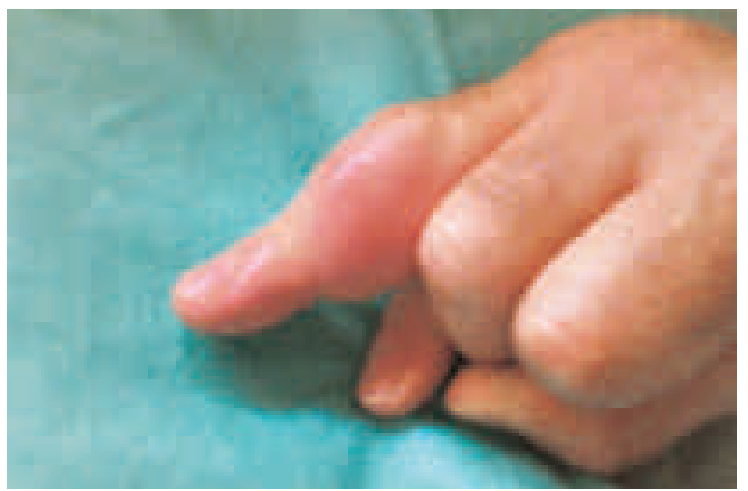

Figure 3 - Typical aspects of dactylitis, involving the second interphalangeal joint of right hand.

and/or non-marginal syndesmophytes, which represent unusual findings of rheumatoid form. Moreover, extra-articular features are a very rare topic. Oligoarthritis is the third frequent subset accounting for $14 \%$ of the cases. It consists of asymmetric involvement of a few joints (less than four) with a typical feature of dactylitis (Fig. 3).

Distal interphalangeal arthritis is the most characteristic feature of psoriatic arthritis, accounting for $5 \%$ of the cases. It was classically described by Charles Bourdillon in 1888 (5). This subset may occur as an exclusive finding, although it complicates all other subsets more frequently.

Finally, we have to consider the uncommon mutilans subset with typical osteolysis of phalangeal or metacarpal bones. 
Table II - Psoriatic arthritis sine psoriasis.

It is not a rare clinical subject (about 20\% of all arthritic cases). It shows a marked occurrence of dactylitis and DIP arthritis. HLA CW6 may be a genetic marker.

\section{PSORIATIC ARTHRITIS "SINE PSORIASIS"}

In this subset (Table II), while articular involvement is clinically expressed, cutaneous is apparently absent. However, a familial history of psoriasis in first or second-degree relatives is a clinically equivalent element, therefore, we have recently focused our attention on this clinical form (6). We know from epidemiological studies that in $20 \%$ of the cases of psoriatic arthritis, articular involvement may precede the onset of skin rash. This condition, therefore, should not be such a rare clinical finding. In our experience, psoriatic arthritis "sine psoriasis" is characterized by a common occurrence of dactylitis and DIP arthritis. In addition, the distribution of HLA haplotypes shows an increased frequency of CW6.

\section{EARLY PSORIATIC ARTHRITIS}

In our view, this subset (Table III) should include patients of established or those of "sine psoriasis" form, whose joint involvement lasts for no more

Table III - Early psoriatic arthritis.

It is characteristically an oligo-enthesoarthritis, with a consistent risk of clinical progression, which occurs in subjects belonging to established or "sine psoriasis" subsets.

Methotrexate, a traditional DMARD, seems ineffective to control all clinical aspects of this syndrome. than twelve weeks (7). The most common clinical presentation is an oligo-enthesoarthritis; polyarthritis and spondylitis are quite uncommon. The distribution of joint involvement shows that enthesopathies, dactylitis and DIP arthritis are characterizing features, particularly in patients with a "sine psoriasis" subset. In our opinion, this form has a consistent risk of clinical progression. In addition, treatment with traditional DMARDs seemed ineffective in controlling all clinical aspects of this syndrome (8).

\section{REFERENCES}

1. Moll JM, Wright V. Psoriatic arthritis. Semin Arthritis Rheum. 1973; 3(1): 55-78.

2. Jones SM, Armas JB, Cohen MG, Lovell CR, Evison $\mathrm{G}, \mathrm{McHugh}$ NJ. Psoriatic arthritis: outcome of disease subsets and relationship of joint disease to nail and skin disease. Br J Rheumatol. 1994; 33(9): 834-9.

3. Scarpa R, Oriente P, Pucino A, Vignone L, Cosentini E, Minerva A, Biondi Oriente C. The clinical spectrum of psoriatic spondylitis.Br J Rheumatol. 1988; 27(2): 133-7.

4. Scarpa R, Biondi Oriente C, Oriente P. The classification of psoriatic arthritis: what will happen in the future? J Am Acad Dermatol. 1997; 36(1): 78-83.

5. Bourdillon C. Psoriasis et Arthropathies. University of Paris: 1888. MDThesis.

6. Scarpa R, Cosentini E, Manguso F, Oriente A, Peluso R, Atteno M, Ayala F, D'Arienzo A, Oriente P. Clinical and genetic aspects of psoriatic arthritis "sine psoriasis". J Rheumatol. 2003; 30(12): 2638-40.

7. Scarpa R, Atteno M, Peluso R, Iervolino S, Di Minno NMD, del Puente A. The Clinical Pattern of early psoriatic arthritis. Rheumatology. 2006; 45 (suppl 1): i65-6.

8. Scarpa R, Peluso R, Atteno M, Di Minno NMD, Iervolino S, del Puente A. Methotrexate for early psoriatic arthritis: results of a pilot study. Rheumatology. 2006; 45 (suppl 1): i67. 